\title{
EMISSÕES DE POLUENTES PROVENIENTES DO TRÁFEGO DE AERONAVES NO AEROPORTO INTERNACIONAL DE SALVADOR.
}

\author{
T. C. BRAGA ${ }^{1}$, E. L. de ALBUQUERQUE ${ }^{1}$ \\ ${ }^{1}$ Instituto Federal da Bahia, DEPEQ. \\ E-mail para contato: taianecbraga@gmail.com
}

\begin{abstract}
RESUMO - Neste estudo, observou-se o tráfego civil de aeronaves no aeroporto Internacional Deputado Luís Eduardo Magalhães em três períodos diferentes, normalizando-se o tráfego para uma base anual, considerando-se cenários diferentes de movimentação. Foram consideradas somente as emissões provenientes do ciclo LTO (landing and take-off) para cada tipo de aeronave, identificando-se os que mais contribuem para a emissão de poluentes e determinando as emissões totais (base anual). Os resultados seguiram aproximadamente a ordem de magnitudes dos fatores de emissão utilizados nas estimativas, sendo o gás estufa $\mathrm{CO}_{2}$ (valor máximo de $1,8 \times 10^{8} \mathrm{~kg}$ por ano) o poluente emitido majoritariamente, seguido de NOx (5,3 x $10^{5} \mathrm{~kg}$ por ano), CO $\left(4,1 \times 10^{5} \mathrm{~kg}\right.$ por ano), $\mathrm{SO}_{2}\left(\left(4,1 \times 10^{4} \mathrm{~kg}\right.\right.$ por ano), NMVOC $\left(3,6 \times 10^{4} \mathrm{~kg}\right.$ por ano), $\mathrm{N}_{2} \mathrm{O}\left(4,7 \times 10^{3} \mathrm{~kg}\right.$ por ano) e $\mathrm{CH}_{4}\left(4,1 \times 10^{3} \mathrm{~kg}\right.$ por ano).
\end{abstract}

\section{INTRODUÇÃO}

O aeroporto Internacional de Salvador Deputado Luís Eduardo Magalhães destaca-se por ser o oitavo aeroporto de maior movimentação do Brasil e maior na região nordeste. Responsável por 4,62\% dos embarques em 2013, 4.241.255 de passageiros embarcados pagos, representando para o mesmo ano um total de 42.130 decolagens (ANAC, 2013). Atividades aeroportuárias contribuem para a degradação da qualidade do ar urbano, pois, além da movimentação propriamente dita de aeronaves, há transporte terrestre de passageiros e cargas, armazenamento de combustíveis, abastecimento de aeronaves, estacionamentos para veículos automotores e fluxo de veículos automotivos em seu entorno. Nestas fontes emissoras são emitidos diariamente diversos gases de efeito estufa (GEE) e outros poluentes do ar, tais como $\mathrm{CO}_{2}, \mathrm{CH}_{4}, \mathrm{~N}_{2} \mathrm{O}, \mathrm{CO} \mathrm{NO}$, $\mathrm{SO}_{2}$, material particulado além de diversos tipos de compostos orgânicos voláteis não metânicos (NMVOC). Neste sentido, há necessidade de se conhecer o inventário de emissões destas atividades para se conhecer o impacto ambiental oriundo destas atividades na qualidade do ar.

No presente estudo, monitorou-se dados recentes de movimentação aeroviária do aeroporto Internacional Luís Eduardo Magalhães. Estes foram empregados para estimar as emissões totais (em base anual) de diversos poluentes atmosféricos em Salvador, contribuindo para o entendimento do papel da movimentação de aeronaves nos impactos ambientais causados por este modal na degradação da qualidade do ar. 


\section{METODOLOGIA}

O método utilizado para a contabilização das emissões totais provenientes das aeronaves (inventário de emissões) empregou a metodologia descrita em IPCC (2006). Em função dos dados disponíveis, optou-se pelo detalhamento conhecido por Tier 2, no qual as emissões totais nas proximidades da superfície podem ser estimadas a partir do número de ciclos landing and take-off (LTO). Estes ciclos abarcam as etapas de voo realizadas pelas aeronaves desde a descida, a partir da altura de cruzeiro, quando da entrada da aeronave na zona de mistura (limite da altura da camada de inversão), incluindo a aterrissagem e o taxiamento até os portões de desembarque. Além destas, também inclui o taxiamento até a pista de decolagem, a decolagem e a subida da aeronave até a saída da zona de mistura, antes de atingir a altitude de cruzeiro (BRASIL, 2010).

No cálculo efetuado, a emissão total de um gás poluente i por uma aeronave do tipo n,

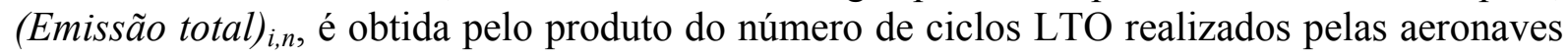
do tipo n, (Número de ciclos LTO) ${ }_{n}$, pelo fator de emissão de cada poluente i por ciclo LTO por tipo de aeronave n, (Fator de Emissão por ciclo LTO) ${ }_{i, n}$, conforme a Equação 1:

$$
\left(\text { Emissão total }_{i, n}=(\text { Número de ciclos LTO })_{n} .(\text { Fator de Emissão por ciclo LTO })_{i, n}\right.
$$

Os fatores de emissão por ciclo LTO foram obtidos em IPCC (2006), onde se considerou GEE $\left(\mathrm{CO}_{2}, \mathrm{CH}_{4}\right.$ e $\left.\mathrm{N}_{2} \mathrm{O}\right)$ e poluentes gasosos (CO, NOx, NMVOC e $\left.\mathrm{SO}_{2}\right)$.

Ao todo, foram monitorados três períodos, compreendendo de uma a quatro semanas: período 1 (de 29/10 a 04/11/2013), período 2 (de 01/11 a 30/11/2013 e período 3 (de 24/11 a 07/12/2014). Esta época do ano (primavera - verão) representa um período de transição entre as estações baixa e alta, de forma que são esperados valores mais próximos da movimentação média real. O número de ciclos LTO por período monitorado foi obtido diretamente do sítio eletrônico da INFRAERO (portal "voosonline"), onde se disponibiliza o monitoramento diário de aeronaves por aeroporto, identificando-o pelo número do voo (INFRAERO, 2014).

Complementarmente, o tipo de aeronave empregado em cada voo foi obtido a partir das tabelas de voos autorizados vigentes (HOTRAN), disponibilizados pela ANAC em seu sítio eletrônico. Obteve-se valores médios para a movimentação anual no aeroporto calculando-se a movimentação média diária em cada período e multiplicando a mesma por 365 dias.

\section{RESULTADOS E DISCUSSÃO}

Na Tabela 1, estão expostos os fatores de emissão obtidos para os diversos tipos de aeronaves que trafegam diariamente no aeroporto Luís Eduardo Magalhães. Observa-se que os mesmos são maiores para o GEE $\mathrm{CO}_{2}$, seguido dos poluentes $\mathrm{NO}_{\mathrm{x}}$ ou $\mathrm{CO}$, a depender do tipo de aeronave. Em menores magnitudes se mostraram os fatores de emissão para os poluentes $\mathrm{NMVOC}$ e $\mathrm{SO}_{x}$, seguidos dos $\mathrm{GEE} \mathrm{N}_{2} \mathrm{O}$ e $\mathrm{CH}_{4}$. Em destaque, estão em negrito os dois maiores fatores de emissão para cada gás pesquisado. Nota-se que, as aeronaves Boeing 767 - 300, A310, A330 - 200 e ERJ - 190 possuem alguns dos maiores fatores de emissão. 
Tabela 1. Fatores de emissão (kg de gás/LTO) para cada tipo de aeronave.

\begin{tabular}{|c|c|c|c|c|c|c|c|}
\hline \multirow{2}{*}{$\begin{array}{c}\text { Tipo de } \\
\text { aeronave }\end{array}$} & \multicolumn{7}{|c|}{ Gases Emitidos } \\
\hline & $\mathrm{CO}_{2}$ & $\mathrm{CH}_{4}$ & $\mathbf{N}_{2} \mathrm{O}$ & $\mathbf{N O}_{\mathrm{x}}$ & $\mathrm{CO}$ & NMVOC & $\mathrm{SO}_{2}$ \\
\hline $737-700$ & $2,5 \mathrm{E}+03$ & $9,0 \mathrm{E}-02$ & $1,0 \mathrm{E}-01$ & $9,1 \mathrm{E}+00$ & $8,0 \mathrm{E}+00$ & $7,8 \mathrm{E}-01$ & $7,8 \mathrm{E}-01$ \\
\hline $737-800$ & $2,8 \mathrm{E}+03$ & $7,0 \mathrm{E}-02$ & $1,0 \mathrm{E}-01$ & $1,2 \mathrm{E}+01$ & $7,1 \mathrm{E}+00$ & $6,5 \mathrm{E}-01$ & $8,8 \mathrm{E}-01$ \\
\hline $757-200$ & $4,3 \mathrm{E}+03$ & $2,0 \mathrm{E}-02$ & $1,0 \mathrm{E}-01$ & $2,3 \mathrm{E}+01$ & $8,1 \mathrm{E}+00$ & $2,0 \mathrm{E}-01$ & $1,4 \mathrm{E}+00$ \\
\hline $767-300$ & $5,6 \mathrm{E}+03$ & $1,2 \mathrm{E}-01$ & 2,0E-01 & $2,8 \mathrm{E}+01$ & $1,4 \mathrm{E}+01$ & $1,1 \mathrm{E}+00$ & $1,8 \mathrm{E}+00$ \\
\hline A310 & $4,8 \mathrm{E}+03$ & 6,3E-01 & $2,0 \mathrm{E}-01$ & $1,9 \mathrm{E}+01$ & $2,8 \mathrm{E}+01$ & $5,7 \mathrm{E}+00$ & $1,5 \mathrm{E}+00$ \\
\hline A319 & $2,3 \mathrm{E}+03$ & $6,0 \mathrm{E}-02$ & $1,0 \mathrm{E}-01$ & $8,7 \mathrm{E}+00$ & $6,4 \mathrm{E}+00$ & $5,4 \mathrm{E}-01$ & $7,3 \mathrm{E}-01$ \\
\hline A320 & $2,4 \mathrm{E}+03$ & $6,0 \mathrm{E}-02$ & $1,0 \mathrm{E}-01$ & $9,0 \mathrm{E}+00$ & $6,2 \mathrm{E}+00$ & $5,1 \mathrm{E}-01$ & 7,7E-01 \\
\hline A321 & $3,0 \mathrm{E}+03$ & $1,4 \mathrm{E}-01$ & $1,0 \mathrm{E}-01$ & $1,7 \mathrm{E}+01$ & $7,6 \mathrm{E}+00$ & $1,3 E+00$ & $9,6 \mathrm{E}-01$ \\
\hline A330 - 200 & $7,1 \mathrm{E}+03$ & $1,3 \mathrm{E}-01$ & 2,0E-01 & $3,6 \mathrm{E}+01$ & $1,6 \mathrm{E}+01$ & $1,2 \mathrm{E}+00$ & $2,2 \mathrm{E}+00$ \\
\hline F100 & $2,4 \mathrm{E}+03$ & $1,4 \mathrm{E}-01$ & $1,0 \mathrm{E}-01$ & $5,8 \mathrm{E}+00$ & $1,4 \mathrm{E}+01$ & $1,3 E+00$ & $7,6 \mathrm{E}-01$ \\
\hline ATR - 72 & $6,2 \mathrm{E}+02$ & $3,0 \mathrm{E}-02$ & $2,0 \mathrm{E}-02$ & $1,8 \mathrm{E}+00$ & $2,3 \mathrm{E}+00$ & $2,6 \mathrm{E}-01$ & 2,0E-01 \\
\hline ERJ - 190 & $2,5 \mathrm{E}+03$ & $1,5 \mathrm{E}-01$ & $7,6 \mathrm{E}-02$ & $6,8 \mathrm{E}+00$ & $1,6 \mathrm{E}+01$ & $1,3 \mathrm{E}+00$ & $7,9 \mathrm{E}-01$ \\
\hline
\end{tabular}

$\mathrm{Na}$ tabela 2 apresentam-se as extrapolações obtidas para as movimentações das aeronaves em um ano de operação (mínima, média e máxima, desvio padrão e coeficiente de variação). Observa-se que os cinco tipos de aeronaves com maior movimentação nestes períodos foram (em ordem decrescente): Boeing 737 - 800, A320, ERJ - 190, ATR - 72, A319 e Boeing 737 - 700. Nota-se ainda que as aeronaves onde houve maior incerteza (imprecisão) nas estimativas da movimentação de aeronaves foram: A310 (125\%), A330 200 (85\%), F100 (76\%), Boeing 757 - 200 (31\%), Boeing 737 - 700 (21\%) e ERJ - 190 $(21 \%)$.

Na Figura 1 estão mostrados, na forma de porcentagens, os resultados da estimativa efetuada para a movimentação anual média de aeronaves no Aeroporto Luís Eduardo Magalhães. Pode-se concluir que as aeronaves mais freqüentemente presentes neste aeroporto são, em ordem decrescente, 737 - 800 (30\%), A320 (28\%), ERJ - 190 (15\%), ATR - 72 (11\%) entre outros presentes em menores percentuais. Estes quatro tipos de aeronaves correspondem a cerca de $84 \%$ do total de aeronaves movimentado no aeroporto estudado. De posse dos fatores de emissão apresentados na tabela 1 e tomando-se os valores mínimo (cenário 1), médio (cenário 2) e máximo (cenário) para o número de aeronaves movimentadas no aeroporto, foram calculadas as emissões totais de cada gás por meio da equação 1 .

Na Figura 2, estão mostrados os resultados obtidos para as emissões totais. Observa-se que as emissões totais seguiram aproximadamente a ordem de magnitudes dos fatores de emissão dispostos na tabela 1. Vê-se que o gás poluente emitido majoritariamente foi o $\mathrm{CO}_{2}$ (valor máximo de $1,8 \times 10^{8} \mathrm{~kg}$ por ano), seguido de $\mathrm{NO}_{\mathrm{x}}\left(5,3 \times 10^{5} \mathrm{~kg}\right.$ por ano), $\mathrm{CO}\left(4,1 \times 10^{5}\right.$ $\mathrm{kg}$ por ano), $\mathrm{SO}_{2}\left(\left(4,1 \times 10^{4} \mathrm{~kg}\right.\right.$ por ano), NMVOC $\left(3,6 \times 10^{4} \mathrm{~kg}\right.$ por ano $), \mathrm{N}_{2} \mathrm{O}\left(4,7 \times 10^{3} \mathrm{~kg}\right.$ por ano) e $\mathrm{CH}_{4}\left(4,1 \times 10^{3} \mathrm{~kg}\right.$ por ano). Estes resultados eram esperados em função da ordem de grandeza dos fatores de emissão dispostos na tabela 1 , sendo os fatores de emissão do $\mathrm{CO}_{2}$ de três até cinco ordens de grandeza maiores que os dos demais. 
XI Congresso Brasileiro de Engenharia Química em Iniciação Científica Unicamp - Campinas - SP 19 a 22 de julho de 2015

Tabela 2. Estimativas para o número total de aeronaves movimentadas no Aeroporto Luís Eduardo Magalhães no período de um ano e estatísticas descritas.

\begin{tabular}{|c|c|c|c|c|c|c|c|c|}
\hline \multirow[b]{2}{*}{$\begin{array}{l}\text { Tipo de } \\
\text { Aeronave }\end{array}$} & \multicolumn{3}{|c|}{$\begin{array}{c}\text { Estimativas para } 1 \text { ano de } \\
\text { operação }\end{array}$} & \multicolumn{5}{|c|}{ Estatísticas descritivas } \\
\hline & $\begin{array}{c}\text { Período } \\
1\end{array}$ & $\begin{array}{c}\text { Período } \\
2\end{array}$ & $\begin{array}{l}\text { Período } \\
3\end{array}$ & 兽 & 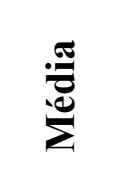 & 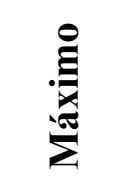 & $\begin{array}{l}\text { Desvio } \\
\text { padrão }\end{array}$ & $\begin{array}{c}\text { Coef. de } \\
\text { variação } \\
(\%)\end{array}$ \\
\hline $737-700$ & 1877 & 2 & 1460 & 1460 & 1859 & 2239 & 3,91 & 21 \\
\hline 737- 800 & 13036 & 13980 & 14313 & 13036 & 13776 & 14313 & $6,4 \mathrm{E}+02$ &, $7 \%$ \\
\hline $757-200$ & 261 & 256 & 130 & 130 & 216 & 261 & $6,7 \mathrm{E}+01$ & $31 \%$ \\
\hline $767-300$ & 52 & 49 & 52 & 49 & 51 & 52 & $1,5 \mathrm{E}+00$ & $3,0 \%$ \\
\hline A310 & 52 & 12 & 0 & 0 & 21 & 52 & $2,6 \mathrm{E}+01$ & $125 \%$ \\
\hline A319 & 2294 & 2531 & 1955 & 1955 & 2260 & 2531 & $2,9 \mathrm{E}+02$ & $13 \%$ \\
\hline $\mathbf{A 3 2 0}$ & 12358 & 12763 & 13479 & 12358 & 12867 & 13479 & $5,6 \mathrm{E}+02$ & $4,4 \%$ \\
\hline A321 & 1095 & 1095 & 1460 & 1095 & 1217 & 1460 & $1,9 \mathrm{E}+02$ & $15 \%$ \\
\hline A330 - 200 & 2451 & 572 & 495 & 495 & 1173 & 2451 & $9,9 \mathrm{E}+02$ & $85 \%$ \\
\hline F100 & 365 & 365 & 0 & 0 & 243 & 365 & $1,9 \mathrm{E}+02$ & $76 \%$ \\
\hline ATR -72 & 4641 & 4696 & 5710 & 4641 & 5016 & 5710 & $5,4 \mathrm{E}+02$ & $11 \%$ \\
\hline ERJ - 190 & 7665 & 7616 & 4875 & 4875 & 6719 & 7665 & $1,4 \mathrm{E}+03$ & $21 \%$ \\
\hline Total & 46146 & 46173 & 43930 & 43930 & 45416 & 46173 & $1,1 \mathrm{E}+03$ & $2,5 \%$ \\
\hline
\end{tabular}

Fonte: Autoria própria.

Figura 1 - Distribuição (em porcentagem) da movimentação média anual de aeronaves no Aeroporto Luís Eduardo Magalhães.

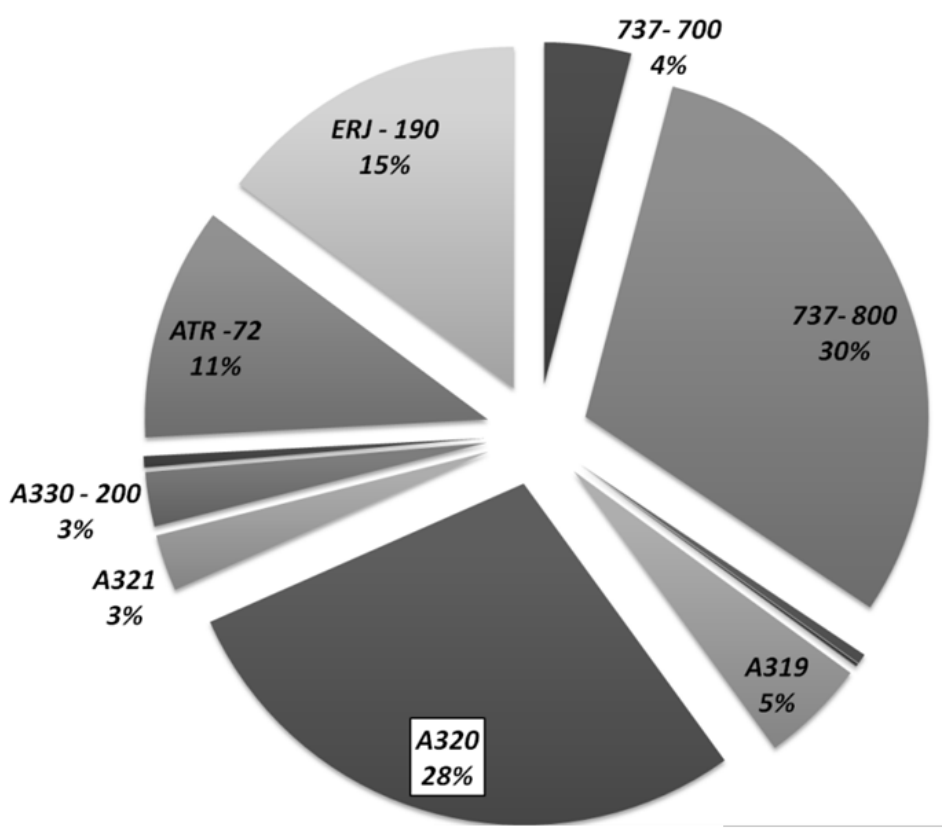




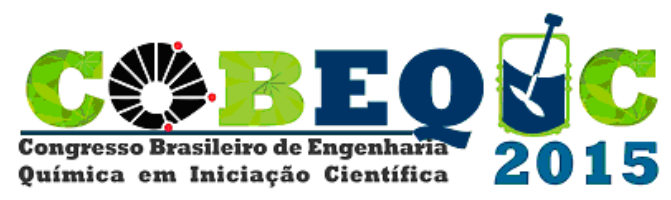

XI Congresso Brasileiro de Engenharia

Química em Iniciação Científica

Unicamp - Campinas - SP

19 a 22 de julho de 2015

Figura 2 - Emissões totais de poluentes no Aeroporto Luís Eduardo Magalhães (kg / ano).

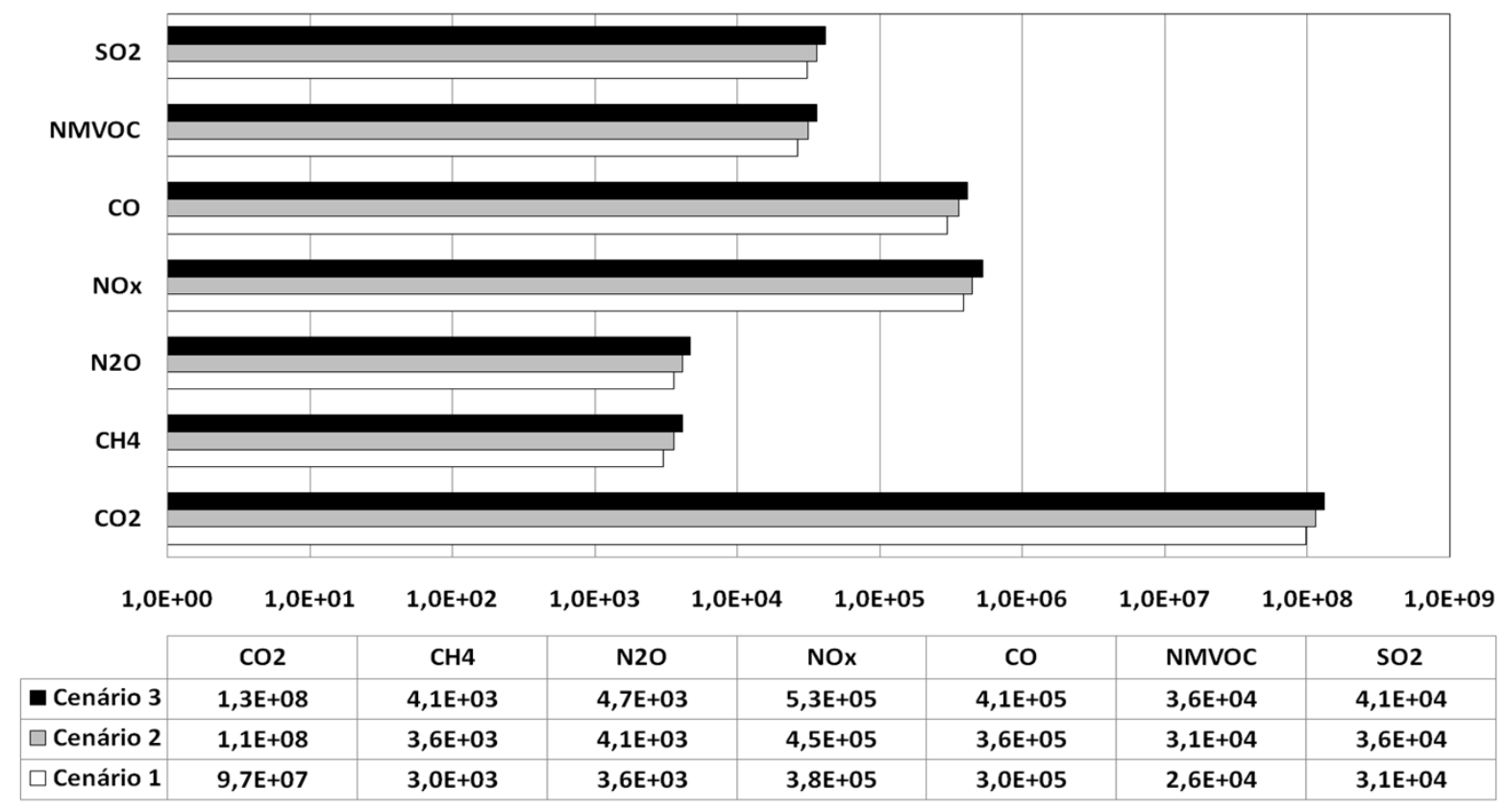

Na tabela 3, estão dispostas as contribuições relativas das aeronaves para as emissões totais de gases poluentes no Aeroporto Luís Eduardo Magalhães, considerando-se o cenário 3 (mais restritivo). As emissões de gases estufa foram calculadas em equivalente de $\mathrm{CO}_{2}$ (eq. em $\mathrm{CO}_{2}$ ), empregando-se 21 e 310 como fatores de conversão, respectivamente, das emissões de $\mathrm{CH}_{4}$ e do $\mathrm{N}_{2} \mathrm{O}$ em eq. em $\mathrm{CO}_{2}$ (IPCC, 2006).

Tabela 3. Contribuição percentual de cada tipo de aeronave na emissão dos poluentes.

\begin{tabular}{|c|c|c|c|c|c|}
\hline \multirow{2}{*}{$\begin{array}{l}\text { Tipo de } \\
\text { Aeronave }\end{array}$} & \multicolumn{5}{|c|}{ Contribuição para a emissão do poluente (\%) } \\
\hline & GEE & $\mathbf{N O}_{\mathbf{x}}$ & $\mathrm{CO}$ & NMVOC & $\mathrm{SO}_{2}$ \\
\hline $737-700$ & 4,0 & 4,0 & 4,0 & 5,0 & 4,0 \\
\hline 737- 800 & 30 & 34 & 25 & 26 & 30 \\
\hline $757-200$ & $<1,0$ & $<1,0$ & $<1,0$ & $<1,0$ & $<1,0$ \\
\hline $767-300$ & $<1,0$ & $<1,0$ & $<1,0$ & $<1,0$ & $<1,0$ \\
\hline A310 & $<1,0$ & $<1,0$ & $<1,0$ & $<1,0$ & $<1,0$ \\
\hline A319 & 5,0 & 4,0 & 4,0 & 4,0 & 5,0 \\
\hline $\mathbf{A 3 2 0}$ & 25 & 23 & 20 & 19 & 25 \\
\hline A321 & 3,0 & 5,0 & 3,0 & 5,0 & 3,0 \\
\hline$\overline{A 330}$ - 200 & 13 & 17 & 10 & 8,0 & 13 \\
\hline F100 & $<1,0$ & $<1,0$ & $<1,0$ & $<1,0$ & $<1,0$ \\
\hline ATR -72 & 3,0 & 2,0 & 3,0 & 4,0 & 3,0 \\
\hline ERJ - 190 & 15 & 10 & 29 & 27 & 15 \\
\hline
\end{tabular}

Fonte: Autoria própria. 
Observa-se que os tipos de aeronaves mais frequentemente encontrados neste aeroporto $(737$ - 800, A320, ERJ - 190 e A330 - 200) são as que mais emitem GEE, respondendo por $83 \%$ das emissões totais destes. Para os poluentes $\mathrm{NO}_{\mathrm{x}}, \mathrm{CO}, \mathrm{NMVOC}$ e $\mathrm{SO}_{2}$, nota-se que as contribuições dos quatro tipo de aeronaves mais presentes no Aeroporto Luís Eduardo Magalhães são semelhantes em seu total, atingindo de 80 a $84 \%$ das emissões totais de cada poluente considerado. Em termos de contribuições relativas, estas são as mesmas para GEEs e $\mathrm{SO}_{2}$, as quais se assemelham às encontradas para $\mathrm{NO}_{\mathrm{x}}$. Nestas, aeronaves dos tipos 737 - 800 e A320 contribuem com 55 a $57 \%$ das emissões totais. As contribuições relativas foram semelhantes para os poluentes $\mathrm{CO}$ e NMVOC, diferindo dos demais poluentes. Naqueles, aeronaves ERJ - 190 e 737 - 800 participam com mais de 50\% das emissões.

\section{CONCLUSÃO}

Nesta pesquisa, realizou-se o inventário de emissões provenientes da movimentação de aeronaves civis no Aeroporto Luís Eduardo Magalhães. Desta forma, identificou-se os tipos de aeronaves que mais circulam na região e estimou-se sua contribuição na emissão de poluentes. Tais resultados mostraram que a ordem de grandeza dos fatores de emissão de cada aeronave foram determinantes para as emissões totais obtidas.

As estimativas realizadas permitem concluir que os poluentes emitidos em maiores quantidades são, nesta ordem, $\mathrm{CO}_{2}, \mathrm{NO}_{\mathrm{x}}, \mathrm{CO}, \mathrm{SO}_{2}, \mathrm{NMVOC}, \mathrm{N}_{2} \mathrm{O}$ e $\mathrm{CH}_{4}$. As aeronaves que mais contribuíram para a emissão de poluentes foram 737-800, A320, ERJ-190 e A330-200, as quais para todos os gases estudados somaram mais de $80 \%$ das emissões totais anuais. Estes resultados são reflexo da combinação do tipo de combustível empregado em cada aeronave (geralmente querosene de aviação), número e tipo de motores e turbinas utilizadas, além de sua eficiência em termos de consumo de combustíveis. Estes fatores estão sendo investigados para entendimento de sua influência mais direta nas emissões, sendo objeto de novos estudos.

Espera-se que os resultados desta pesquisa auxiliem o poder público e órgãos responsáveis pelo controle do tráfego civil de aeronaves no entendimento dos impactos causados por este modal na qualidade do ar das cidades brasileiras.

\section{REFERÊNCIAS}

ANAC - Agência Nacional de Aviação Civil. Anuário estatístico do transporte aéreo de 2013. ANAC, 2013.

BRASIL. MCT - Ministério de Ciência e Tecnologia. $2^{a}$ Comunicação Nacional à Convenção Quadro das Nações Unidas sobre Mudança do Clima, v.2, 2010.

INFRAERO - Empresa Brasileira de Infraestrutura Aeroportuária. Consulte seu voo. Disponível em: < http://voos.infraero.gov.br/voos/index.aspx >. Acessado em: novembro de 2014.

IPCC - Intergovernmental Panel on Climate Change. Guidelines for National Greenhouse Gas Inventories, Institute for Global Environmental Strategies. Japan, 2006. 